\title{
END TO END IP QOS ASSURANCE USING POLICY BASED MULTI-AGENTS SLA MANAGEMENT SYSTEMS
}

\author{
Mauro Fonseca and Nazim Agoulmine
}

\begin{abstract}
The development of Internet as well as overall communication technologies has created a rery competitive communication market. In fact. there is no doubt that in the near future. every company and perhaps every person in the world will have an physical or wireless Internet access via an ISP (Internet Service Provider). Thus, the Internet became a very complex global network which represents an opportunity to provide worldwide value-added services requiring a certain level of quality of service (QoS) such as QoS-VPN (Virtual Private Network), VoD (Video On Demand). telelearning, e-Commerce, etc. Thus, we propose in this work to extend this approach in order to integrate new mechanisms that will allow customers to request end-to-end services. This assurance covers the QoS as well as associated paranteters such as cost. The proposed solution introduces interoperability mechanisms between ISP policy based management systems and uses mobile agents to facilitate the negotiation between domains. The agent negotiation process is constrained by a set of predefined policies in order to limit the boundaries of the SLA parameters such as the cost. QoS, etc.
\end{abstract}

Keywords: SLA, QoS, ISP, Management, Policy, Agent technology, Interoperability, Negotiation.

Resumo - A evolução da Internet bem como de todas as tecnologias dé comunicação tem desenvolvido un mercado muito competitivo. Na realidade, caminhamos para un futuro onde todas as empresas e talvez todas as pessoas no mundo terão acesso à Internet via un ISP (Intemet Service Provider). Assim sendo, a Internet se torna uma complexa rede global que representa uma oportunidade para prover serviços em escala mundial, necessitando garantias de níveis de qualidade de serviços. Nós propomos neste trabalho melhorar esta metodologia para integrar novos mecanismos que permitirão aos usuários finais a requisição da garantia de serviços fim à fim. Estas garantias são ligadas à Qualidade de Serviço (QoS) e aos parâmetros associados à faturação. A solução proposta introduz mecanismos de interoperabilidade entre um sistema de gerenciamento baseado em políticas (PBM) pertencentes ao ISP e agentes móveis pertencentes aos usuários para facilitar a negociação entre diferentes domínios. O processo de negociação é conduzido por um conjunto de políticas pré-definidas para limitar os valores dos parâmetros do SLA como custo, QoS etc.

Palavras-chave: SLA, QoS, ISP, Gerenciamento, Política, Tecnologia de Agentes, Interoperabilidade, Negociação.

Mauro Fonseca and Nazim Agoulmine are with the LIP6 and LSC Laboratories, France (E-mails: (Mauro.Fonseca@lip6.fr, Nazim.Agoulmine@lip6.fr)

\section{INTRODUCTION}

The Internet became at the center of all communications between end users. customers and companies. It is clear that IP will be at the heart of many future services such as VoD, e-commerce, e-learning, etc. Internet service providers are thus companies seeking new way of doing business. While at the first time, the objectives was to provide more and more bandwidth to attract customers and allow them to use freely bandw idth consuming services, the recent results have shown that this approach was not necessarily the more efficient one as many customers were not satisfied by the provided service. In fact, it is recognized that end-users do not have the same expectation from the network. Some are only interested by low level QoS applications such as emails and are not willing to pay a lot while others, request high-level QoS for business applications, VoD, etc and are ready to pay a higher price. The approach that is gaining more approval now is the differentiation between customers so that the provided service is not the best one but the one that corresponds closely to the end users' requirements. Service Providers are therefore investigating opportunities to providing differentiated SLA (Service Level Agreement) to their Customers to identify the terms of agreements concerning the quality of each provided service from the requested source access point to the destination access points. Quality of Service can cover many aspects of the relationship and not only the performance, for instance it includes aspects such as service provisionning time, customer care, maintenance, security, billing, etc. These agreed terms are all grouped and identified in the SLA (Service Level Agreement).

Nowadays, many ISPs have already start to apply this strategy in their domain and the SLA became a differentiation factor between them. From the customer's perspective, the main choice criteria is the capacity of the provider to fulfil its contractual responsibilities as defined in the SLA and to respond to its future requirements. The first aspect is usually well satisfied as operators have a better knowledge about users' satisfaction and perception of QoS. In fact, in each domain, ISPs have gained experience in the translation of customers' service demand into operational performance parameters. This is particularly true because the operator has a complete control over its physical equipments and then can configure them to satisfy as much as possible its customers' requirements. However, this necessitates that the customers' access point are reachable from the ISP POP (Point of Presence). The customers can control the provided service using monitoring information usually provided by the operator itself.

However. when the customer destination access points are not all in the ISP domain, the process became more complex. In fact, the ISP is not able to control the end-to-end behavior 
of the provided service and need to collaborate with peer ISPS to define the special treatment to apply to services that span their respective domain. Nowadays, this process is realized using fax and telephone and necessitates a long delay-time to allow the administrators to verify the agreed terms and to set up the network elements configuration. The complexity of approach reduces the possibilities of agreements and increase the risks of customers' unsatisfaction due to the perturbation of their services.

The objective of this paper is to investigate the possibility to automate this process so that the negotiation can be achieved in a shorter time without any human implication. Each ISP specifies its high-level business policy in term of collaboration rules with other ISPs to provide services spanning a number of administrative domains. We suppose that each ISP has already installed a management system that is able to configure (and monitor) network equipments to provide certain classes of service (mainly we are concerned with DiffServ classes). ISPs can define different collaboration strategies to offer end-to-end SLA to their customers.

The complex aspects of this negotiation are the various parameters to consider as well as their semantic in each domain. For instance, a gold service in one domain could be different from a gold service in another one. Thus, when an ISP negotiate with its customer an end-to-end gold service, it has to verify whether the customer traffic will be served as a gold service from ingress point to egress point even if the service go through a different domain. So if gold services from peer domains are not equivalent in term of QoS with the local gold service, then ISP has to find out a solution to maintain the initial agreed level of service. One solution will be to upgrade the requested service in the peer domain, i.e. ask for a premium service instead of a gold service in the domains where gold services have lower QoS than the local gold service.

The remainder of the paper is organized as follows: section 2 describes the background concepts for the purpose of this work. Section 3 presents the objectives of this work. The following section presents the proposed framework for interdomain policy based management using mobile agents. Section 5 describes the architectures of the different components of the framework. And finally a conclusion and intended future works.

\section{BACKGROUND CONCEPTS}

\subsection{POLICY BASED MANAGEMENT}

The main input for this work is the result of the Policy Working Group [1] of the Internet Engineering Task Force that has accomplished a lot of work in this area. This group has defined a scalable and secure framework for policy definition and administration [2] [3]. The framework defines a set of components to enable policy rules definition, persistent storage and enforcement [4].

The first component, as showed in Figure 1. is the Policy Enforcement Point (PEP). It is a policy decision enforcer component installed in the network policy enabled equipments. The second component is the Policy Decision Point

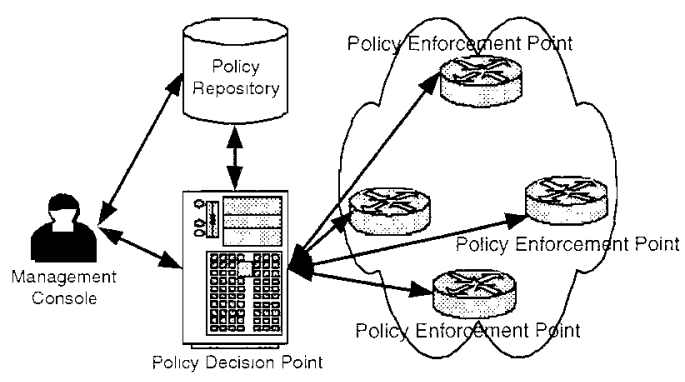

Figure 1. Policy based management model.

(PDP) that is the decision-making component. The administrator has access to the PBM system through the management console. It is the GUI that allows the definition and the maintenance of policies. These later are defined according to the high level strategy of the organization as well as the various SLA established with end-customers. Policy based managenient systems require a global information model to capture all information needed to perform policies i.e. information about the network equipments, the services, the customers, etc.

Different models have been proposed such as the DMTF (Desktop Management Task Force) called CIM (Common Information Model).

The CIM is an extensible model that captures every notion that is applicable to all areas of management : system, network and users information as well as policy definition [5]. It is an implementation neutral schema for describing overall management information in terms of objects instances, properties, relationships, classes, and subclasses.

It extends the existing instrumentation and management standards such as IETF/SNMP, DMTF/DMI, ISO/CMIP, using object-oriented constructs and design. DEN another initiative, which is an ad-hoc group of DMTF, has worked out on a specification of management using directories. It has mainly defined an information model for network elements and services as well as its representation in LDAP. This work has strongly influenced the CIM definition.

\subsection{SERVICE LEVEL AGREEMENT (SLA) AND SERVICE LEVEL SPECIFICATION (SLS)}

SLA is a formal negotiated agreement between two parties, sometimes called a Service Level Guarantee. It is a contract that exists between the Service Provider and the Customer, designed to create a common understanding about services, priorities, responsibilities, etc. [3]

The technical parameters agreed between the two parties are specified in a sub-part of the SLA called SLS (Service Level Specification). It specifies how the customers' traffic will be treated by the provider network [6]. From the operator perspective. individual metrics have to be identified and enforced in the network in order to provide the customers with the agreed SLA. Hence monitoring mechanisms should be activated to control the behavior of the network. These tasks and parameters are called Service Level Objective (SLO) [6]. 


\subsection{AGENT TECHNOLOGIES}

Mobile agent technology is now a recognized concept for distributed systems management [7]. In fact. mobile agents are widely used to solve problems encountered in large scale distributed and real-time systems where the volume and com- - iexity of the interactions make it difficult to implement classical client-server solutions.

Generally, an agent can be considered as an assistant or helper, which performs routine and complex tasks on the user"s behalf. In the context of distributed computing, an agent is an autonomous software component that acts asynchronously on the user's behalf. Agent types can be broadly categorized as static or mobile [8] [9].

The main motivation of using this technology is driven by the desire to automate the control and management processes related to policy negotiation between various administrative domains while simplifying the interaction model by delegating some responsibilities to agents [10] [11] [12].

\section{OBJECTIVE OF THIS WORK}

Nowadays many ISPs have provided a QoS IP infrastructure allowing new type of services such as: IP voice. Video, Virtual Private Network, etc. However, the services are only provided in the ISP domain. In fact, it has been found to be very complex to deploy these services upon a set of multiprovider domains. When deploying such services in this context, the configuration tasks became very complex because of the difference in operators' strategies and the heterogeneity of their underlying network technologies.

This difficulty is exacerbated by the fact that each provider nas its own service definition (premium. gold, silver, etc) and consequently complicating the definition of the end-to-end service. Nowadays, these tasks are mainly performed through human interactions using faxes and telephone calls between the various organizations and manual configuration.

This approach introduces high-level misconfiguration risks as well as long delay time for service provisioning. Thus, the : iea of this work is to automate the negotiation and configiration procedures so that to allow rapid end-to-end service introduction with negotiated SLA.

The starting point of the overall process is a customer willing to use a service spanning several administrative domains. Each domain belongs to a particular ISP that has its own objectives and strategy but willing to cooperate with other ISPS to offer these end-to-end services with negotiated SLA. The ISPs possibly use heterogeneous underlying network technologies, nevertheless, we assume that each organizational domain has deployed a Policy-Based Network Management System playing the role of Bandwidth Broker (BB) for its domain and thus providing a uniform interface to configure the underlying network.

Based on these assumptions, the objective is to enhance the $\mathrm{BB}$ with capabilities allowing the interaction with peer BB to negotiate local and multi-providers SLA. The interaction will be based on a set of predefined agreements established between the various ISP in the I2I SLA (ISP to ISP SLA). These agreements are the formal negotiated terms between an
ISP Provider and an ISP Customer for service delivery. It is designed to create a common understanding about services, priorities. responsibilities, etc between the ISPs. Similarly, end-users can establish a SLA with a particular ISP for the provisioning of an end-to-end service which characteristics are captured in the C2I SLA (Customer to ISP SLA).

When the service requested by a customer span a number of ISPs ' domain, a negotiation process has be launched between ISPs in order to set up the customer service from end to end For instance, if the customer requests a service spanning several domains, the initiating ISP has to search for the various routes to the destination customer access point. If different routes are possible, then the process should identify the best solution according to the customer preferences in term of QoS, Price or any other aspect defined in the requested SLA. Thus, it is necessary to enhance the PBM framework in order to take into account the multi-party process of policy based management as described in the Figure ?.

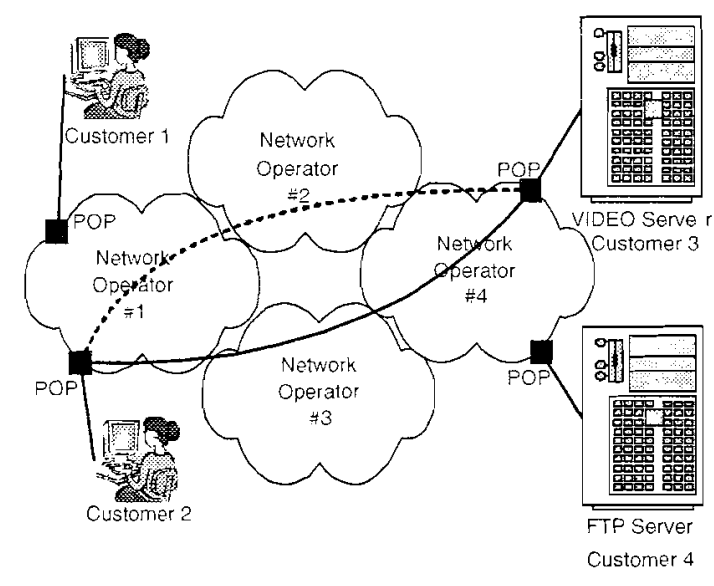

Figure 2. End-to-end service spanning multiple domains.

ISP2ISP SLA and C2ISP SLA are conceptually the same but different in term of realization as they do not address the same level of service granularity and time scale. In fact, the C2ISP SLA has a fine granularity of service definition while the ISP2ISP SLA has a wide granularity. This means that the negotiation process between ISPs is usually none frequent and based on a large amount of bandwidth for each negotiated service while the negotiation between a customer and its ISP is usually more frequent and concerns smaller amount of bandwidth for each requested service.

The automation between ISP PBM systems will hide the complexity of the end-to-end negotiation process. In fact, the different ISPs can have different agreements with each others to provide connectivity to the same destination, thus permitting a competitive market to take easily place.

PBM approach will facilitate the representation of ISP strategies through policy rules however it will not facilitate the automation of the negotiation process itself. For this reason, we propose to use mobile agents as a flexible approach to introduce PBM over multi-domain IP networks.

The motivation to use mobile agents is the fact that negotiation between the customer and the ISP and an ISP and another ISP may be very complex and it will be higher facilitated if some kind delegation is realized to reduce the high 
number of interactions if a classical client server protocol is used. Hence, the philosophy of policy based management will help this approach as agent can themselves carry business policies so that the negotiation and the decision can be performed locally.

\section{NEGOTIATION OF QOS PARAMETERS}

In order to understand the problem, we have taken the topology presented in the Figure $\mathbf{2}$ as the main use case for this work. This figure shows four ISPs willing to cooperate in order to provide end-to-end services. We have completed it with information at the network level. Let suppose that the ISP1 is implementing the following quality of service strategy defining 3 classes of service:

- Gold: Traffic in this category is allocated 50 percent of the available bandwidth.

- Silver:Traffic in this category is allocated 30 percent of the available bandwidth.

- Bronze: Traffic in this category is allocated 20 percent of the available bandwidth.

The three service are defined using the following QoS parameters : delay, jitter, packet loss and throughput which affect the customer"s traffic and are part of the negotiated SLA. At this point, we didn't take into account, for simplification reasons, other parameters of the SLA as described in [13].

If the customer requests a service between his site a remote site, the delay that the customer's applications will see is the end to end latency introduced all along the network path due to queuing, processing or congestion in each intermediate node. In our case, we have to deal with the multiple domains networks behavior. In this case the global latency is additive, i.e.

$\mathcal{D}_{\text {end2end }}=\sum_{i=1}^{n} \mathcal{D}_{i}$

The jitter is the distortion of the inter-packet arrival times compared to the inter-packet times of the original transmission (i.e. delay variance). Jitter is particularly damaging to multimedia traffic. In the case of a connection spanning multiple domains delay variation accumulates on an RMS basis, i.e.

$\mathcal{D} V_{\text {tot }}=\sqrt{\left(D T_{1}+D V_{2}^{2}+\ldots+D V_{n}\right)} ;$

where $\mathrm{Dn}$ is the mean one-way delay of Domain $\mathrm{n}$ and $\mathrm{Vn}$ is the standard deviation of the delay variation of Domain $n$.

The loss is the failure of a transmitted packet to be received. usually because it was dropped at some point along the network path due to congestion. Thus when spanning multiple domains the loss probability accumulates on a probabilistic basis, i.e.

$\mathcal{L P}_{\text {tot }}=1-\left[\left(1-I P_{1}\right) \times\left(1-I P_{2}\right) \times \ldots \times\left(1-L P_{n}\right)\right]:$

Let suppose that the objective of the ISPI is to maintain the previous service in the following boundaries. The way the
ISP divides its bandwidth and how it distributes it between different classes is independent from the requirements of the customers. However, the ISP has to assess his choices in the middle term according to the network utilization and the QoS failure in the network (monitoring loop).

- Gold :

$$
\begin{aligned}
& \text { - DSCP : EF } \\
& \text { - delay : Max }=10 \mathrm{~ms} \\
& \text { - jitter : Max }=1 \mathrm{~ms} \\
& \text { - packet loss : Max } 10^{-12} \\
& \text { - throughput : } 500 \mathrm{Mbps}
\end{aligned}
$$

- Silver :

$$
\begin{aligned}
& \text { - DSCP : AF } \\
& \text { - delay : Max }=20 \mathrm{~ms} \text {, Probability }=10^{-3} \\
& \text { - jitter: Max }=5 \mathrm{~ms}, \text { Probability }=10^{-3} \\
& \text { - Packet loss: } \operatorname{Max} 10^{-6}, \text { Probability }=10^{-3} \\
& \text { - throughput : } 30 \mathrm{Mbps}
\end{aligned}
$$

- Bronze :

$$
\begin{aligned}
& \text { - DSCP : BE } \\
& \text { - Throughput : } 20 \mathrm{Mbps}
\end{aligned}
$$

If these services have to be provided from end to end, the ISP can't assure that all crossed networks in the path to the destination POP will assure these services. Thus, there is a need to collaborate with remote ISPs in order to identify the locally available services and their corresponding QoS and define the corresponding rules.

As presented, we have introduced two types of SLAs: The Customer to ISP SLA (C2I SLA) and the ISP to ISP SLA (I2I SLA).

\subsection{C2ISP SLA SPECIFICATION}

The SLA between the customer and the ISP specifies the following information: 1)who is the customer, 2)what service he is willing to have, 3 )when he is willing to use it, 4)from where he is going to use it (and how to monitor the provided service for billing purpose and cash back in case of failure). The four units that describe this SLA are introduced in the Internet draft [14] and presented in the following:

\section{- Common agreements:}

- Description of the customer/provider/service:

- Time validity period (permanent or at certain date/time):

\section{- Topology agreements:}

- Service Access Points

- Graph describes the type of connection the customer is going to set up: 1-1 or 1-M or $\mathrm{M}-1$ or $1-*$ or $*-1$. etc. At this point we consider only a point to point services. 


\section{- QoS agreements:}

- Traffic descriptor

- Load descriptor

- QoS parameters

- The fourth is the monitoring unit including parameters concerning the monitoring of the service which is not yet considered in this work.

Traffic descriptor describes the packet streams of the customer for which the QoS unit attributes apply. This can be a DSCP, a TCP or UDP Source Port, a Destination Port. a Protocol, a Layer2Specification.

Load descriptor describes the type of load the customer is going to send or receive as well as the treatment to none conform traffic. These information will allow the PBM system to $:=i$ up the right policing of the traffic at the customer ingress access routers. Excess traffic can be dropped. shaped or remarked.

The main parameters are :

- Delay unit :

- Max Delay to be seen by each conforming packet.

- Max Probability that this max delay is not respected,

- Loss unit

- Max Loss Probability defining the experience of end-to-end loss of conforming packets,

- Max probability that this Max Loss is not respected.

- Jitter unit

- Max Jitter seen by each conforming packet (Mean Jitter seen by conforming flow)

Based on these information. we have upgraded the domain information model with an object class representing the C2I SLA. It is mainly composed by a set of attributes representing relationships to other object classes such as customer class. time validity period classes, SAP classes, Type of graph class. QoS unit class.

As example of an agreed SLA. mainly based on the work introduced in [14], we presents a customer 1 willing to set up a video conference service with a customer 2 located in a remote geographical position in the same operator network Some enhancements to the draft work are introduced to highlight the QoS parameters in the SLA.

The instance object model for this example is presented in the following Figure 3 .

We didn't consider billing information in this initial model ¿r simplification reasons but this important aspect will be sonsidered in future works.

\section{REQUIRED INFORMATION MODELS}

When a service spans a number of ISPs domains, a negotiation process between ISPs has to be launched in order to control the end-to-end behavior of the service.

This negotiation process can be performed in two different ways:

In this first approach called synchronous approach, ISPs that are willing to cooperate initiate a negotiation. This process allows the exchange of capabilities information between the ISPs i.e. service definitions in each ISP domain (premium. gold. silver. etc). the minimum bandwidth allocation per service for inter ISP links, the usage cost of this service, available POPs, etc. Thus. each time a customer requests a new service. the associated ISP has aiready the knowledge about the various peer ISPs" services, QoS and cost to take a decision. When a ner allocation is realized with a remote ISP. inter-domain resource usage and resources allocation information are maintained. This approach necessitates a regular update of information between the ISP so that any changes in one domain can be notified to other domains. For example, if one ISP changes its service cost, it has to notify the changes to the peer ISPs.

An other approach, called asynchronous approach set up a central ISP information repository that contains information about all existing ISPs. ISPs do not have any a priori knou?edge about other ISPs. When a service is requested, the customer defines its service access points (SAP). If the destinations SAPs are outside the ISP domain, then a request is sent to the central repository to search the list of ISPs to which the customer SAPs are connected to. Then, the initiating ISP negotiates with the peer ISPs an end-to-end service. This scenario is similar to what a trader does for distributed applications in an Open Distributed System. The central repository functionality can be provided by a specific VASP (Value Added Service Provider).

Both approaches are interesting, however we have focused in this work on the first approach that means that the initiating ISP knows exactly what are his peer ISPs to the destination SAPs.

\subsection{INTER DOMAIN INFORMATION MODEL}

The initial interaction between ISPs permits to identify what are the reachable networks across these ISPs domains (this can be also learned implicitly through an external routing protocol such as BGP or explicitly through the negotiation protocol) and what type of services each ISPs is offering (qualitative information such as voice, video, data, premium. gold, silver, bronze).

In order to maintain theses information in the information model of the ISP, we have added new object classes such as:

Peer ISP class : represents the peer ISP that could be part of the service chain to the remote access point. It contains all the information that identify the remote ISP, its Points of Presence (POP) and exchange points (EP) with other ISPs.

Peer ISP service class : represents a description of the portfolio services provided by the remote ISP. This class permits to maintain detailed information about the quality of service provided by a remote ISP as well as its properties (cost. 


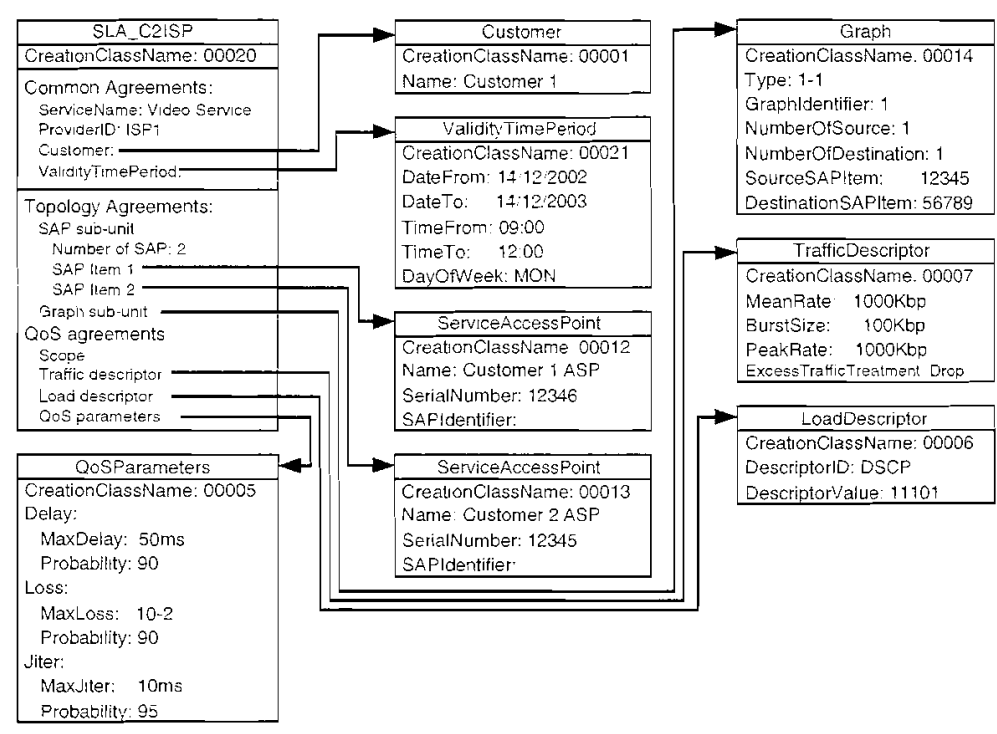

Figure 3. OO Specification of the Customer to ISP Service Level Agreement (I2C SLA).

security, etc) for each service.

Peer ISP SLA : represents inter-domain SLA between the local ISP and the peer ISP. It represents the agreed terms between two ISPs concerning the delivery of a particular service. This SLA is very important when monitoring the quality of service of the provided service and identifying any violation of agreed contractual parameters.

\subsection{ISP2ISP SLA SPECIFICATION MODEL}

An SLA between ISPs has an aggregate structure comparing to the SLA between a customer and an ISP. As previously explained, the main difference concerns the level of granularity of a service an ISP can request from a peer ISP. For example, a customer can request a DSO service to an ISP, however an ISP can ${ }^{\circ} t$ ask less than a NxTl service, $N$ is defined by the ISP providing the inter-ISP service. The $\mathrm{N}$ parameter is then a negotiation parameter that could influence the choice of the remote ISP for the delivery of the end-to-end service.

In this case, each ISP has to maintain a resources model that represents the resources that are available in inter-ISP links to remote peer ISP networks. Each time an ISP receives a request for a new service from a customer, it has to verify whether there is enough resources at the time of activation of the requested the service to the destination domain. Thus a forecast model should be defined and maintained in each domain to calculate during time the allocation and utilization of resources in the inter-domain communications.

The proposed ISP to ISP SLA is shown in Figure + ran instance of a Video service trunk SLA is represented).

\section{PROPOSED ARCHITECTURE}

After the definition of the various models necessary to maintain information about the resources and SLA. we propose in the following an agent architecture to help the realization of the negotiation process between the various domains. The idea is to allow the agents to have enough autonony to negotiate the best service on the behalf of the customers or ISPs depending on their predefined strategy.

The ISP SLA management system is also based on a set of agents howerer these are static agents performing local operations. The agent approach permits to decompose the management system into components that can be easily combined with the underlying PBM components. The PBM system allows the effective control and configuration of the ISP' network equipments to reflect the high level agreements as shown in Figure 5.

- SLA subscription (SSU) agent : this agent is responsible for the interactions with the customer agent to subscribing a new SLA. As a result it creates an C2I SLA object in the common information model as well as related objects.

- Inter-domain SLA subseription (ISSU) agent: this agent is responsible for the processing of all SLA negotiation with peer ISPs. It uses an ISP agent to interact with remote ISPs. As a result it creates I2I SLA objects in the common information model as well as related objects.

- SLA Admission Control (SAC) Agent this agent is responsible to:

- Interact with the $\mathrm{CN}$ agent for the verification of the terms the new SLA with the available resources in the domain and between domains.

- Get arailable resources in the domain with NRAM agent.

- Get arailable resources in the inter domain with INRAM agent.

- Create and sends new C2ISP SLA objects as well as related objects to NRAM agent.

- Create and sends new I2I SLA objects as well as related objects to INRAM agent. 


\begin{tabular}{|c|c|c|c|c|}
\hline Common agreements: & & & & \\
\hline 1- Provider Id: & ISP 1 & & & \\
\hline 2- Customer ld: & Customer 1 & & & \\
\hline 3- Service name: & Video Senicâ & & & \\
\hline 4- Time Stamp: & $01203+55 \%$ & & & \\
\hline & Time From: & $109: 00$ & & \\
\hline & Time To: & $12: 00$ & & \\
\hline 5- Time Validity Period & Date From: & 14062002 & & \\
\hline & Date To: & $140^{7} 2003$ & & \\
\hline & Days of the Week: & MONDAY & & \\
\hline Topology agreements: & & & & \\
\hline & Number of SAP & 2 & & \\
\hline & & Seriai number: & 12345 & \\
\hline & SAP item 1 & $600^{2}$ & Type: & iFV4 Address \\
\hline 6- SAP sub-unit & & SAP Identifler: & Value: & $190.20 .20 .1 / 24$ \\
\hline & & Seriai number: & 6789 & \\
\hline & SAP item? & & Type: & |FV/4 Address \\
\hline & & ISAP identifier: & Value: & 200.20 .20 .224 \\
\hline & Type: & $1-1$ & & \\
\hline & Graph indentifíer: & 1 & & \\
\hline & Number of Source: & 1 & & \\
\hline 7- Graph sub-unit & Number of destination: & 1 & & \\
\hline & Source SAP tem: & Serial number: & 12345 & \\
\hline & Destination SAP item & Serial number: & 6789 & \\
\hline QoS agreements: & & & & \\
\hline & Graph identifier: & 1 & & \\
\hline 8. Scope: & Trallic descriotor: & DSCP: & 11101 & \\
\hline & Mean rate: & $500 \mathrm{~Kb} / \mathrm{s}$ & & \\
\hline 0 L od d corinto. & Burst size: & $1000 \mathrm{~Kb}$ & & \\
\hline 9- Load descriptor: & Peak rate: & $1.5 \mathrm{Mb} / \mathrm{s}$ & & \\
\hline & Excess Traffic Treatement: & DROP & & \\
\hline & & Max Dejay: & $50 \mathrm{~ms}$ & \\
\hline & Delay: & Probability: & $\begin{array}{l}90 \\
\end{array}$ & \\
\hline & 1058. & Max Loss: & $10-2$ & \\
\hline 10- QoS parameters & Loss: & Probability: & 90 & \\
\hline & 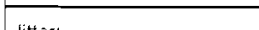 & Max sitter & $10 \mathrm{~ms}$ & \\
\hline & Jitter: & Probability: & 90 & \\
\hline & Service availabilit;:": & $100^{\circ}{ }_{0}^{\circ}$ & & \\
\hline Cost agreement: & & & & \\
\hline 11- Fixed cost: & & 1.700 Unit/Mbp & iminute & \\
\hline 12-Variable cost: & & & & \\
\hline SLA Violation: & & & & \\
\hline
\end{tabular}

Figure 4. ISP2ISP SLA.

- Process all SLA negotiation requests from peer ISPs. It uses an IN agent to interact with remote ISPs.

- Network resource allocation model (NRAM) agent : this agent keeps an up to date information about the available resources in the ISP network as well future resources allocation.

- Inter-domain network resource allocation model (INRAM) agent : this agent keeps an up-to-date information about the available resources between peer ISPs network as well as the future allocation of resource.

- Policy generator (PG) agent : it main role is to translate accepted SLA into operational policies and store them into the CIM.

- Customer negotiation ( $\mathrm{CN})$ agent : is an agent that is instantiated by the customer for the purpose of negotiating end-to-end SLA with its ISP.

- ISP negotiation (IN) agent : is an agent that is insiantiated by an inter-domain SLA agent for the purpose of negotiating end-to-end SLA with the peer ISP.

- The Policy Decision Point (PDP) : is the decisionmaking component that takes as input policies that are stored into the CIM and takes the configuration decisions contained in the action part of the triggered policy rules.
- Common Information Model Repository Agent : It is the CIM object manager. The CIM is the database that contains the instance of the CIM classes and assure the persistency of the PBM system.

\subsection{INTERACTION PROTOCOL BETWEEN A CUSTOMER AND AN ISP}

In the case where the service requested by the customer can be fulfilled directly inside the ISP domain, the interaction protocol between a customer and is based ISP is based on agent interactions only inside the ISP domain.

In order to represent these interactions, we have used the approach proposed in [5]. The SLA negotiation protocol is composed of 6 services : Submit. Refuse, Accept, Propose and Cancel that are self explained in the Figure 6.

The interaction diagram in the Figure 6 shows the scenario where the customer delegates to its agent the authorization to negotiate locally any proposal from the ISP that is different from the initial request. The customer specifies the maximum and minimum boundaries for the negotiated SLA parameters as well as a priority in the negotiation process between these parameters.

Simple rules can be used to represent this policy :

\footnotetext{
$<\mathrm{IF}><$ Proposed $>$ SLA $<$ /Proposed $>$ !=

$<$ Requested $>$ SLA $<$ Requested $>$

$<$ THEN $>$ start negotiation $</$ THEN $></$ IF $>$
} 


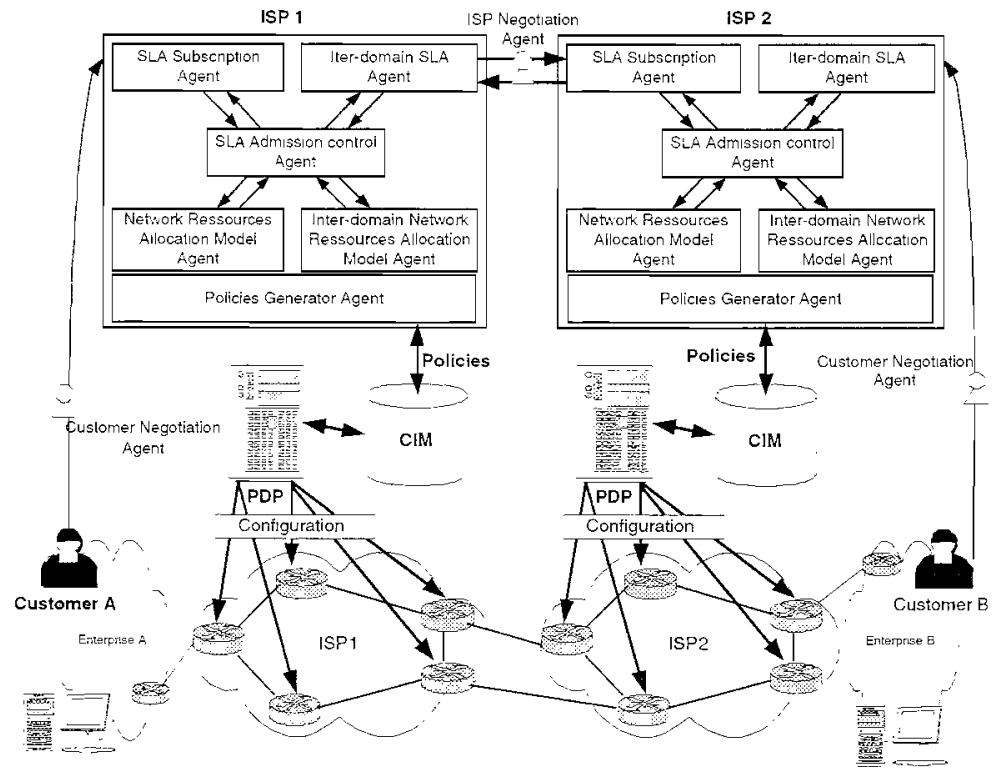

Figure 5. Agent Based Architecture for SLA Management.

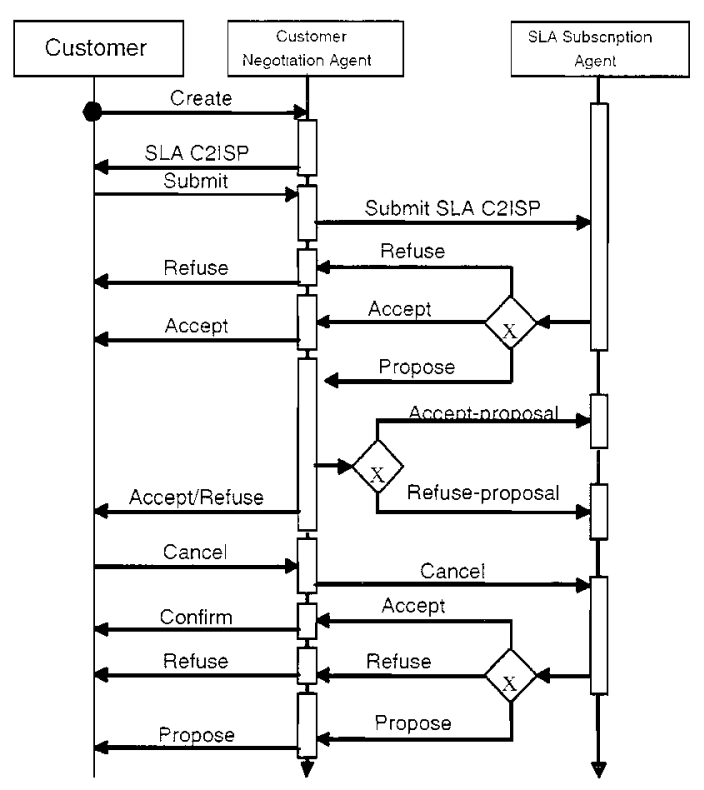

Figure 6. Customer agent and SLA subscription agent protocol specification.

\footnotetext{
// agent is delegated the negotiation process
}

Or,

$$
\begin{aligned}
&<\mathrm{IF}><\text { Proposed }>\text { SLA }<\text { /Proposed }>\text { ! }= \\
&<\text { Requested }>\text { SLA }<\text { /Requested }> \\
&<\text { THEN }>\text { stop negotiation }</ \text { THEN }></ I F>
\end{aligned}
$$

// agent is not allowed to negotiate and terminates the process. Or.

$$
\begin{gathered}
<\mathrm{IF}><\text { Proposed }>\text { SLA }<\text { /Proposed }>\text { ! }= \\
<\text { Requested }>\text { SLA }</ \text { Requested }> \\
<\text { THEN }>\text { request sonice }<\text { THEN }></ \text { IF }>
\end{gathered}
$$

// agent is not allowed negotiate. the final decision is taken by the source.

In case of a local negotiation, further rules can be specified such as:

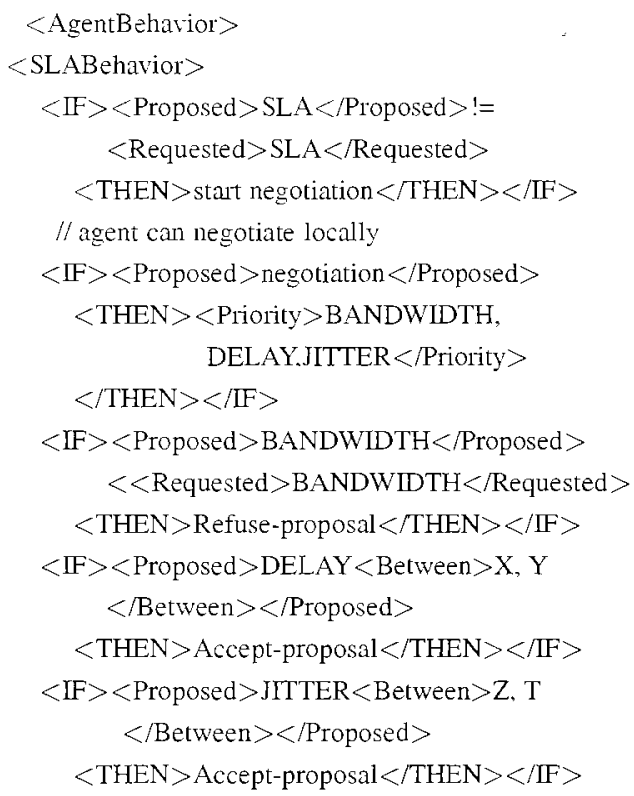

\subsection{INTERACTION PROTOCOL BETWEEN TWO ISPS}

If the ISP is not capable to satisfy the end-to-end terms of the requested service in case where the service span a number of ISP domains, i.e. the requested SAPs are not all internal, the negotiation process became more complex. In fact, the interaction protocol between an ISP SLA management system and a peer ISP should be triggered to identify the possible routes with the requested QoS to the destination SAP belonging to remote ISPs. This process is launched in two cases : when there is no previous allocation of inter-ISP resources or when the available resources for inter-ISPs communications 


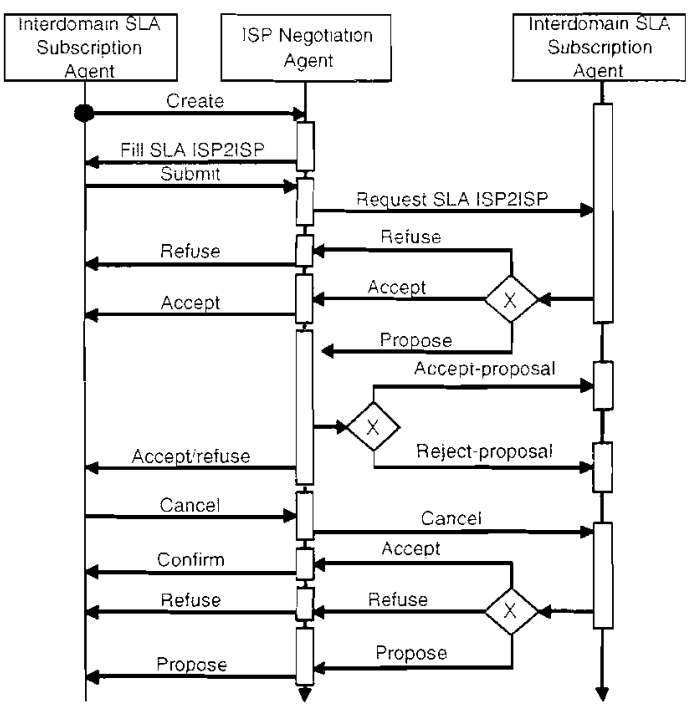

Figure 7. negotiation protocol between an ISP agent and an inter-domain SLA subscription agent.

are sufficient to fulfill the request.

Similarly to the previous negotiation process, the interactions between ISP domain necessitate almost the same phases, as showed in Figure 7. However, the ISP can not negotiate the exact requested values for SLS parameters as the remote ISP will not for small allocation of resources for inter-ISPs commumications but rather on aggregate, Thus the ISP has to define the strategy to delegate to its negotiation agent. For instance, the ISP can fix different constraints rules concerning the maximum cost per Mb it would like to pay, the boundaries for the allocated bandwidth, jitter and loss probability, the maximum time to service (MTTS), etc. Hence, the agent should specify to the remote ISP whether, the local domain will be used a stub domain or as a final domain. If it is a stub domain, this means that the final SAP is in a different network otherwise it is directly connected to the ISP domain.

To express these information in a neutral manner to avoid heterogeneity in data representation between domains. the requested SLA is represented in XML. Thus we have defined new tags to define the agent strategy when negotiation with the peer ISP domain.

\subsection{NETWORK RESOURCE ALLOCATION MODEL}

The network resources allocation model agent is responsible for maintaining an up-to-date model of temporal resources usage. In fact, when an ISP accept an SLAs, it has to activate in a permanent manner or at a certain date/time/duration the corresponding service. Thus, we have defined an agent that is responsible to maintain this model so that to be able to control the acceptance or rejection of new reservation requests according to the available resources.

In this work, we presents one way to maintain this resources model using tables. We suppose that the ISP has initially setup a set services (presented here as $\mathrm{P}$ for premium, $S$ for silver and $B$ for bronze). Each service has a certain amount of initial allocated bandwidth. When SLA are agreed with a customer or a remote ISP, the available resource of the corresponding service decrease

The aims of the various tables is to represent a forecasting model to take into account services that are not activated in a permanent manner. A more efficient approach would have been the use of a symbolic approach however it is more complex to define. In this allocation tables, we suppose that the minimum reservation slot time is 1 hour. Each time an SLA is agreed to provide a service at a certain date/time/duration, its corresponding slot is marked.

Thus. we have specified one table for each service between peer SAPs in the ISP domain. This table is associated with Nx365 tables that define for each day of the year the resources allocation schedule for each agreed SLA (we suppose that the ISP allows reservation for $\mathrm{N}$ complete years with a slicing table approach to eliminate the old year and take into account the new one). see table I.

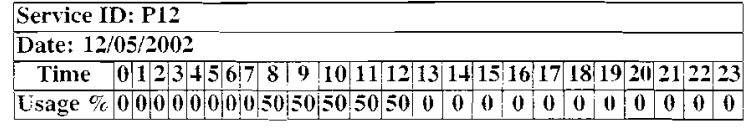

Table 1. Premium service table.

In Table 1, we have represented a schedule for a premium service on the 12/05/2002 starting at 8:00 AM and finishing at 12:00 AM with a reserved bandwidth of 50\% between SAP1 et SAP2.

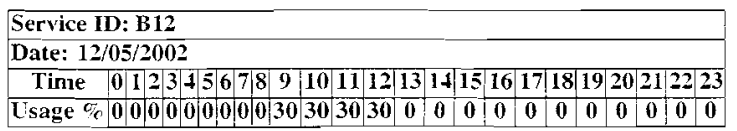

Table 2. Bronze service table.

The Table 2 shows the reservation of $30 \%$ of the bandwidth allocated to the Bronze service between SAP1 and SAP2. The ISP is able to accept any future reservation request as far as the service bandwidth allocation is not fully used.

The same process apply to inter-domain resource model and corresponding agent.

\section{POLICIES GENERATION}

When an SLA is accepted, the SLA subscription agent transfers the decision to the policy generator agent that creates the corresponding policy rules into the CIM. These policies are by the PDP in order to enforce the corresponding configuration in the ISP network through the various PEP.

The policies are defined in term of rules as described in [15]. The policy rules are created according to the accepted SLA terms. They are expressed in term of condition part and action part. When the condition part is verified, the action part is executed. These policies are represented in the CIM using three main classes as follow :

IF $\{$ VendorPolicy Condition $==$ True AND

Polic $y$ TimePeriodCondition $==$ True ,

THEN (execute (Vendor PolicyAction)). 
Based on the agreed SLA terms, several rules are created as follow:

Rule 1 - concerning the ISP commitment for the service delivery. Two parameters are important, 1)the TimeValidityTime and 2)the Agleed QoS. Thus the rule can be expressed as:

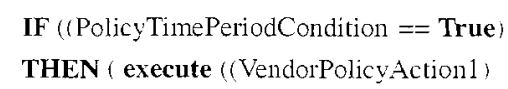

VendorPolicyActionl is transformed by the PDP into a more precise action object according to the underlying technology. For example, in the case of DiffServ, the action classes will be mapped to DiffServ configuration actions. VendorPolicyAction? can be a simple packet accounting action at the interface in case of a flat cost or a complex action in case of a multi-valued billing schema (i.e. session duration, amount of exchanged data. etc).

Rule 2 - concerning the Customer commitment to send a traffic of a certain profile. The rule that constraint the Customer traffic will be defined as follows:

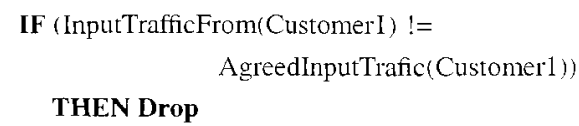

At this stage of the work, we have only address simple rules but more work is necessary to address all the possible cases.

\section{PROTOTYPE IMPLEMENTATION}

The developed prototype for this system is based on Java as programming language, ObjectSpaceTM Voyager ORB 3.0 [16] as the mobile agent platform. DiffServ over Linux [17] and OpenLDAP-2.0.23 [18]. DiffServ over Linux is used to specify various classes of services in the ISPs domain, the OpenLDAP server is used to implement the common information model and finally Voyager ORB is used to develop the multi-agents SLA management system as well as the customer negotiation agent.

\section{CONCLUSION AND FUTURE WORK}

In our proposal, we have introduced several aspects of negotiation between a customer and an ISP and between two ISPs. We have proposed a multi-agents architecture to facilitate these interactions in a flexible and dynamic manner. The multi-agents system is built on the top of a policy based management system that controls the behavior of an ISP network. The customer specifies the QoS it requests from its ISP in terms of a C2I SLA. Among other information, this SLA de- fines the maximum acceptable price the customer is willing to pay for that service. The customer delegates to an agent the negotiation process with the peer ISP which decides whether to accept or reject the request.

Different interactions strategy between the customer agent and the ISP have been defined depending on the level of delegation provided by the customer to its agent. When a service spans a number of admimistrative domains, a negotiation process is launched between ISPs in order to satisfy the hop-byhop assurance of the requested service. Each ISP specifies at a high level its strategy in term of policy rules and let the multi-agents system automatically decide whether to accept or reject the new request according to the available resources and what cost to apply.

This approach has shown an interesting starting results and many other results are expected.

As future works, we are working on the mapping between the SLA level and the network level as it has been realized in a trivial manner in the first prototype. It is also planned to study in a more detailed manner the impacts of the ISPs' strategy on the global QoS and pricing in an open telecom market.

\section{REFERENCES}

[1] P. F. Group, "Description of working group," Policy Framework Group, IETF, 2002. www.ietf.org/html.charters/policycharter.html.

[2] R. A. P. Group, "Description of working group," Resource Allocation Protocol Group, IETF, 2002. www.ietf.org/html.charters/rap-charter.html.

[3] Stardust.com, "Qos protocols \& architecture." QoS Fortun White Paper, july 1999. www.qosforum.com.

[4] S. Blake and A1., "An architecture for differentiated services," Network Working Group, IETF, vol. RFC 2475. December 1998. www.ietf.org.

[5] J. O. Bernhard Bauer, Jörg P. Müller, "Agent uml: A formalism for specifying multiagent interaction," Agent-Oriented Software Engineering, pp. 91-103, 2001. Springer.

[6] A. Westerinen and Al.. "Terminology for policy-based management." Network Working Group, IETF, vol. RFC3198, November 2001.

[7] M. Breugst and T. Magedanz, "Mobile agents-enabling technology for active intelligent network implementation," IEEE Network Magazine - Issue on Active and Programmable Networks, vol. 12, May 1998.

[8] H. S. Nwana and M. Wooldridge, "Software agent technologies," BT Technology Joumal, vol. 14, pp. 68-78, October 1996.

[9] D. C. et al., "A mobile agent brokering environment for the future open network marketplace," Seventh International Conference On Intelligence in Services and Networks (IS\&N2000), February 2000.

[10] N. A. Allan Marshall, "An agent-based paradigm for managing service quality in open network environments," Multimedia Management. August 2002.

[11] A. Marshall. "Dynamic network adaptation techniques for improving service quality," Networking 2000, May 2000.

[12] N. Agoulmine and Al., "Trouble management for multimedia services in multi-provider envi-ronments." Intemational Journal on Network and Service Management, vol. 8, pp. 99-123, January 2000. Wiley publisher. 
[13] M. Smirnor and al. "Sla networks in prenium ip," $C A$ DENUS, january 2001. "ww.cadenus.org/deliverables/d11final.pdf.

[14] S. D. R. Rajan. E. Celenti. "Service level specification for inter-domain qos negotiation," Internet Draft, November 2000. draft-somefolks-sls-00.txt.

[15] Stardust.con, "Introduction to qos policies," QoS Forum White Paper. Septembre 1999. ww.qosforum.com.

[16] I. Recursion Softwate. "Voyager." 2002. www.recursionsw.com.

[17] sourceforge. "Differentiated services on linux." may 2001. diffserv.sourceforge.net.

[18] O. Foundation, "Open ldap." May 2001. www:openldap.org/software/.

Mauro Fonseca is a PhD student in LIP6 Laboratory at University of Paris VI. His research interest includes Mobile Agent, Policy Based Management. Active Networking and Nework management. He received the B.Eng in computer engineering from PUCPR, Brazil and M.S. in electronic engineering from CEFET-PR. Brazil.

Nazim Agoulmine is a Full Professor at the University of Erry. His research interest includes network and service management. policy based management. agent technology, active networks. ad hoc networks. Since 1988, he has participated in a number of European and French research programs in the area of networking. He is also a member of several IEEE and IFIP international conference technical conmittees 